\title{
Method of forest fire automatic early warning and alarm system design
}

\author{
Yucun Wang ${ }^{1}$, Fengyun $\mathrm{Ma}^{2,}$, and Hao Fan $^{1}$ \\ ${ }^{1}$ Shandong Agricultural University School of Information Science and Engineering, Tai'an City, \\ Shandong Province, China. \\ ${ }^{2}$ Shandong Agricultural University School of forestry, Tai'an City, Shandong Province, China.
}

\begin{abstract}
Forest is a huge green wealth bestowed by nature to mankind, but forest fire will cause great harm. Automatic forest fire early warning and alarm system can warn the high fire risk areas in the forest, or find and alarm the forest fire as early as possible, which is of great significance to reduce the loss of forest fire. This paper introduces a construction method of forest fire automatic early warning and alarm system, which provides knowledge and experience accumulation for the subsequent research and development of related systems.
\end{abstract}

\section{Introduction}

Forest is the largest terrestrial ecosystem on earth and an important part of the global biosphere. It is the gene pool, carbon pool, water pool and energy pool on the earth. It plays a vital role in maintaining the ecological balance of the whole earth. It is the resource and environment for human survival and development. Forest is known as the "lung of the earth". Forest is a huge green wealth bestowed by nature. By the end of the Eighth National Forest Resources Inventory, the physical stock of forest resources assets in China was 16.074 billion cubic meters; and the total value of forest land resources assets was 21.29 trillion CNY. The harm of forest fires is amazing. Taking 2016 as an example, China had 2034 forest fires in 2016, with a loss of 60846 cubic meters of forest stock, 137.65 million young trees, and other losses caused by forest fires converted into 41.36 million CNY. ${ }^{[1]}$ Therefore, it is of great significance to build an automatic forest fire early warning and alarm system to warn the high fire risk areas in the forest, or to find and alarm the forest fire as early as possible, which is of great significance to protect forest resources and reduce the loss of forest fire.

\section{Overall system structure}

The automatic forest fire early warning and alarm system consists of on-site information acquisition subsystem, information transmission subsystem and information processing center subsystem. The system's structure is shown in Figure 1. The input of the system

\footnotetext{
Corresponding author: sdmfy@sdau.edu.cn
} 
comes from various sensors of the field data acquisition subsystem, and the output of the system is fire warning signal.

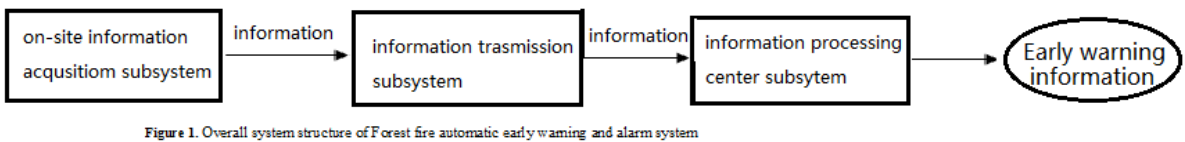

Fig. 1. Overall system structure of Forest fire automatic early warming and alarm system

\subsection{Field information acquisition subsystem}

The occurrence of forest fire is related to the moisture content, temperature, humidity, oxygen concentration and other factors of combustibles in the forest ${ }^{[2]}$, while the spread of forest fire is related to the types of combustibles, terrain, wind speed and other factors ${ }^{[3][4]}$. The on-site information acquisition subsystem is the subsystem that completes the relevant information data acquisition for the early warning and alarm system of forest fire. Using the sensor network composed of various sensors, the on-site information acquisition subsystem can collect various relevant data in the forest fire monitoring area in real time, and transmit the data to the information processing center subsystem through the information transmission network, so as to provide data support for the forest fire early warning and alarm of the information processing center subsystem. The system structure of the field information acquisition subsystem is shown in Figure 2.

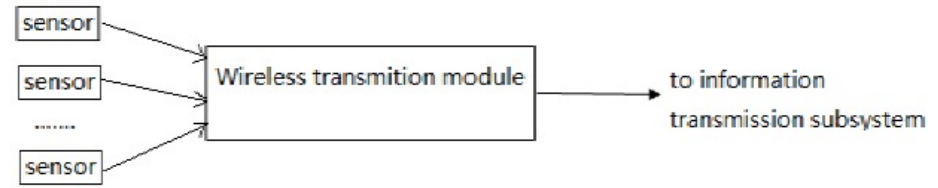

Fig. 2. Field information acquisition subsystem

\subsection{Information transmission subsystem}

The information transmission subsystem completes the information transmission task in the forest fire automatic early warning and alarm system. Since there is no controllable equipment capable of extinguishing forest fires so far, the system does not consider the control of on-site execution equipment, that is, the system does not design the control information transmission from the subsystem of information processing center to the site, Therefore, the subsystem of forest fire automatic early warning and alarm system is only responsible for transmitting various sensing data collected by the on-site information collection subsystem to the subsystem of information processing center in an appropriate information transmission mode. Considering the influence of various factors, the information transmission subsystem mainly adopts $4 \mathrm{G}$ or GPRS to complete the information transmission. For forest areas not fully covered by $4 \mathrm{G}$ or GPRS, satellite communication can be considered to complete the function of information transmission subsystem if conditions permit.

\subsection{Information processing center subsystem}

The information processing center subsystem is the information processing center and decision-making center of the whole system. The information processing center subsystem receives various field data information transmitted by the field information collection subsystem through the information transmission subsystem, analyzes and processes the data 
through a certain algorithm, studies and judges the forest fire risk level of the forest area where each sensor node is located, and gives the corresponding information early warning. If the subsystem of the information processing center studies and judges the occurrence of forest fire for the information transmitted from the sensor, the subsystem of the information processing center shall issue an emergency fire alarm accordingly, and give specific fire geographic location information and more reasonable forest fire extinguishing plan, so as to provide time conditions for extinguishing forest fire as soon as possible. In order to complete the above functions, the subsystem of the information processing center shall not only receive the sensing information, but also locally maintain the database containing meteorological information, historical fire disaster information, topographic information of each forest area, fire source information and other information.

\section{Future prospects and implication of this system}

The development and application of this system provides a new technical means for discovering possible forest fire hazards as soon as possible, and is of positive significance to reduce the fire impact on forest resources. The effective combination of image recognition technology, UAV technology, ${ }^{[5]}$ infrared thermal imaging technology and the system will further improve the performance of the system.

\section{Conclusion}

Forests provide rich green wealth for human society, protect a large number of animal and plant resources, conserve water and soil, and maintain the self balance of the atmosphere on which human beings depend. To prevent and reduce the loss of forest fire as much as possible is not only to protect forest resources themselves, but also to protect human living environment and human beings themselves. Therefore, it is of great significance for the control of forest fire. During the research and development of the system, many specific problems to be solved in forest fire early warning and prediction have been encountered, and rich relevant knowledge and experience have been accumulated, which provides knowledge and experience accumulation for the subsequent research and development of relevant systems.

\section{Acknowledgments}

In the process of writing this article, the author has received the help and guidance of many experts and scholars. I would like to express my heartfelt thanks. In addition, a lot of network information has also been of great help to the author. Much thanks to them too. This study is supported by Shandong Agricultural Science and Technology Fund Project (2019ly004).

\section{References}

1. J. Huang, S. Lee, J. Zhang, et al. J. Fujian agri. Fore. Univ, 50,1(2021).

2. R. Liu, H. Duan, X. Wu, J.Anhui Agric.Sci. 49,4(2021)

3. H. Bai, D. Liu, S. Niu, et al. J. Beij. Fore. Univ, 43,5(2021)

4. H. Hu, Fore. fire ecology manag. M(2005)

5. S. Liu, W. Yao, Y. Zhang, Comp. Engi. Sci. 42,7(2020) 